\title{
Statistical Modeling of Pretilt Angle Control on the Homogeneous Polyimide Surface as a Function of Rubbing Strength and Baking Temperature
}

\author{
Hee-Jin Kang, Jung Hwan Lee, Jeoung-Yeon Hwang, IlGu Yun, and Dae-Shik Seo ${ }^{a}$ \\ Department of Electrical and Electronics Engineering, Yonsei University, \\ 134 Shinchon-Dong, Seodaemun-Gu, Seoul 120 - 749, Korea \\ ${ }^{a}$ E-mail : dsseo@yonsei.ac.kr
}

(Received February 21 2006, Accepted March 24, 2006)

\begin{abstract}
In this paper, the response surface modeling of the control of the pretilt angle in the nematic liquid crystal on the homogeneous polyimide surface with different surface treatment is investigated. The pretilt angle is one of the main factors to determine the alignment of the liquid crystal display. The pretilt angle is measured to analyze the variation of the characteristics on the various process conditions. The rubbing strength and the hard baking temperature are considered as input factors. After the design of experiments is performed, the process model is then explored using the response surface methodology. The analysis of variance is used to analyze the statistical significance and the effect plots are also investigated to examine the relationship between the process parameters and the response.
\end{abstract}

Keywords : Response surface model, Pretilt angle, Rubbing strength, Hard baking temperature, Effect plots

\section{INTRODUCTION}

In recent years, the liquid crystal displays (LCDs) have been extensively used in information display devices such as notebook computers, desktop monitors, televisions and car navigation systems. LCDs require the monodomain alignment and the proper pretilt angle. The pretilt angle prevents the creation of the reverse tilted disclination in the twisted nematic (TN) mode. The uniform director orientation of the liquid crystal (LC) molecules and the control of the pretilt angle on the substrate surfaces are the most important task to improve the performance of LCDs. The generation of the pretilt angle in nematic liquid crystal (NLC) on rubbed polyimide (PI) surface for homogeneous alignment has been discussed by many researchers[1,2]. Presently, a rubbing process is widely used in aligning the LC molecules on the alignment layers.

However, the statistical modeling of the control of the pretilt angle in NLC on the rubbed PI surface for the homogeneous alignment is not reported yet. Very few attempts have been made at the statistical modeling for the pretilt angle. The statistical modeling of the control of the pretilt angle is very useful and gives reliability to predict the pretilt angle. That is the reason why the statistical modeling in the pretilt angle research is important.
The methodology to characterize process using the response surface model with the design of experiments (DOE) has been applied to various fields. As an example, May et al. investigated the statistical experimental design in plasma etch modeling[3]. Garling et al. presented enhancing the analysis of variance (ANOVA) technique to wafer processing[4]. Lau et al. reported Taguchi design of experiment for wafer bumping by stencil printing[5]. Hu et al. optimized the hydrogen evolution activity on zinc-nickel deposition using experimental strategies by statistical methodology[6].

In this paper, the control of the pretilt angle in the NLC on the homogeneous PI surface is characterized by the response surface models. The input factors were explored via D-optimal design with 13 runs. The experiments are performed with two conditions for the rubbing strength and the hard baking temperature. The D-optimal design is used to make design matrix in this experiment efficiently. The relationship between the response and the factors is statistically analyzed by the ANOVA and the effect plots.

\section{EXPERIMENTAL}

The glass substrates are prepared at room temperature $\left(22{ }^{\circ} \mathrm{C}\right)$. The polymer (JSRJALS-1371-R1) is used as a 


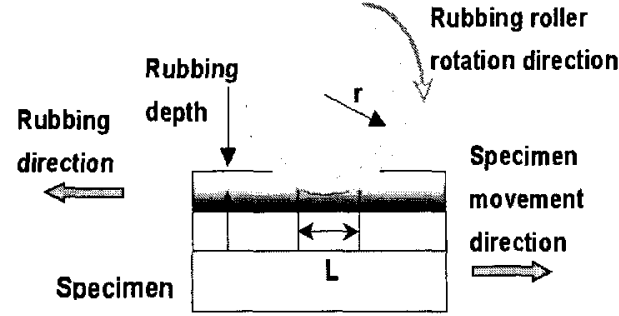

Fig. 1. The schematic of the rubbing machine.

Table 1. The summary of the process conditions.

\begin{tabular}{|c|c|}
\hline Process variable & Range \\
\hline Pre-baking temperature & $80^{\circ} \mathrm{C}$ \\
\hline Hard baking temperature & $180{ }^{\circ} \mathrm{C} \sim 250{ }^{\circ} \mathrm{C}$ \\
\hline Cooling temperature & $22{ }^{\circ} \mathrm{C}$ \\
\hline Measure temperature & $22{ }^{\circ} \mathrm{C}$ \\
\hline Rubbing strength & $0.3 \sim 0.7 \mathrm{~mm}$ \\
\hline Homogeneous polyimide & JSRJALS $-1371-\mathrm{RI}$ \\
\hline Cell gap & $60 \mu \mathrm{m}$ \\
\hline Liquid crystal & $\left(\mathrm{T}_{\mathrm{C}}=72{ }^{\circ} \mathrm{C}, \Delta \varepsilon=8.2\right.$, Merck Co. $)$ \\
\hline
\end{tabular}

homogeneous alignment layer and coated uniformly on the indium-tin-oxide (ITO) electrodes by a spin-coating machine[7-9]. The thickness of the PI film is set at 500 $\AA$. The PI substrates are pre-baked at $80{ }^{\circ} \mathrm{C}$ for 10 minutes. The PI substrates are imidized at $180^{\circ} \mathrm{C}, 215^{\circ} \mathrm{C}$ and $250^{\circ} \mathrm{C}$ for 1 hour, again. These substrates are cooled at room temperature. After that, the substrate surfaces are rubbed by the rubbing machine with the nylon roller ( $\mathrm{Y}_{0}-15-\mathrm{N}$, Yoshikawa Chemical Industries Co.). The rubbing strength has been defined in some previous works[10-12]. The apparatus of the system is schematically shown in Fig. 1.

We use three kinds of the rubbing strengths to make different pretilt angle such as $0.3 \mathrm{~mm}, 0.5 \mathrm{~mm}$ and 0.7 $\mathrm{mm}$. These numerical values represent the rubbing depth closely related to the rubbing strength. The substrates are arranged as a sandwich type for the pretilt angle measurement, and the cell gap is $60 \mu \mathrm{m}$. The fabricated cell is filled with a NLC $\left(\mathrm{T}_{\mathrm{C}}=72^{\circ} \mathrm{C}, \Delta \varepsilon=8.2\right.$, from Merck Co.).

In addition, the pretilt angle is measured using the crystal rotation method at room temperature $\left(22^{\circ} \mathrm{C}\right)$ with TBA-701 made by autronic-MELCHERS GmbH. The process variables and conditions are listed in Table 1.

\section{MODELING SCHEME}

Two input factors are explored via D-optimal Design with 13 runs to minimize the variance associated with the coefficient estimates for the model. This design tends
Table 2. The D-optimal design matrix.

\begin{tabular}{|c|c|c|}
\hline Run & $\begin{array}{c}\text { Rubbing } \\
\text { strength }(\mathrm{mm})\end{array}$ & $\begin{array}{c}\text { Hard baking } \\
\text { temperature }\left({ }^{\circ} \mathrm{C}\right)\end{array}$ \\
\hline 1 & 0.3 & 180 \\
\hline 2 & 0.3 & 180 \\
\hline 3 & 0.5 & 250 \\
\hline 4 & 0.5 & 215 \\
\hline 5 & 0.3 & 250 \\
\hline 6 & 0.7 & 180 \\
\hline 7 & 0.3 & 250 \\
\hline 8 & 0.7 & 250 \\
\hline 9 & 0.5 & 180 \\
\hline 10 & 0.7 & 215 \\
\hline 11 & 0.5 & 215 \\
\hline 12 & 0.3 & 215 \\
\hline 13 & 0.7 & 250 \\
\hline
\end{tabular}

to put most design points on the edges of the design space. All experimental runs are made in random order. The experimental design matrix of input factors used in each run is summarized in Table 2. Quadratic model is used as basic model. The Quadratic model having intercept, two main effects, two square effects, and one two-factor interaction is defined as the following:

$$
Y=\beta_{0}+\beta_{1} X_{1}+\beta_{2} X_{2}+\beta_{3} X_{1}^{2}+\beta_{4} X_{2}^{2}+\beta_{5} X_{1} X_{2}
$$

Where, $Y$ is the response value, $\beta_{i}$ are the model coefficients, and $X_{i}$ are the process factor values.

In order to build the model, the insignificant effects in this study are eliminated backward elimination method under the statistical significance level is $0.01(\alpha=0.01)$. The backward elimination method starts with the equation in which all effects are included and the insignificant effects in the model are eliminated one at a time. At any step, the variable with largest p-value, as computed from the current regression, is eliminated if this p-value exceeds a specified value[13]. The square effect for the rubbing strength is eliminated from the model, because of its significance $(P$-value $=0.353$ ) in the full quadratic model. The selected significant effects are summarized in Table 3.

The analysis of variance for the response is summarized in Table 4. P-value of the main effects, the square effect and the two-factor interaction are 0.001 , 0.008 and 0.007 , respectively. It indicates that all the effects are significant in this model for the pretilt angle. The adjusted R-squared value is 0.971 in Table 5. It means that $97.1 \%$ of variation is being explained by the model. 
Table 3. The summary of the statistical significance.

\begin{tabular}{|c|c|c|}
\hline Response & Effect & p-value \\
\hline Pretilt angle & RS & $<0.0001$ \\
\cline { 2 - 3 } & HBT & $<0.0001$ \\
\cline { 2 - 3 } & HBT $^{*}$ HBT & 0.0080 \\
\cline { 2 - 3 } & RS $^{*}$ HBT & 0.0071 \\
\hline
\end{tabular}

Table 4. The ANOVA for the pretilt angle.

\begin{tabular}{|c|c|c|c|c|c|c|}
\hline Source & DF & Seq SS & Adj SS & Adj MS & F & P \\
\hline Regression & 4 & 103.406 & 103.406 & 25.85149 & 102.6 & 0 \\
\hline Linear & 2 & 97.602 & 8.65716 & 4.32858 & 17.18 & 0.001 \\
\hline Square & 1 & 2.566 & 3.09922 & 3.09922 & 12.3 & 0.008 \\
\hline Interaction & 1 & 3.238 & 3.23803 & 3.23803 & 12.85 & 0.007 \\
\hline $\begin{array}{c}\text { Residual } \\
\text { Error }\end{array}$ & 8 & 2.016 & 2.01568 & 0.25196 & & \\
\hline Lack-of-Fit & 4 & 2.013 & 2.01303 & 0.50326 & 759.64 & 0 \\
\hline Pure Error & 4 & 0.003 & 0.00265 & 0.00066 & & \\
\hline Total & 12 & 105.422 & & & & \\
\hline
\end{tabular}

Table 5. The summary of statistics.

\begin{tabular}{|l|l|l|l|}
\hline Std. Dev. & 0.501957 & R-Squared & 0.98088 \\
\hline Mean & 6.182308 & Adj R-Squared & 0.97132 \\
\hline C.V. & 8.119245 & Pred R-Squared & 0.953642 \\
\hline PRESS & 4.88714 & Adeq Precision & 28.35847 \\
\hline
\end{tabular}

The regression model for the pretilt angle is the following expression:

Pretilt Angle $=-3.655748891-32.74291925 * R S+$

$0.262209494 * H B T-0.000871074 * H B T^{*} H B T+$

$0.100279503 * R S * H B T$

Where, the RS is the rubbing strength and the HBT is the hard baking temperature.

The modeling result exhibit a good agreement with the values between the predicted and the measured response as shown in Fig. 2.

There are three assumptions to use ANOVA, which is independence, normally distributed, and homogeneity of variance. First assumption is that each of the group is independent. Second assumption is that the values in each group of the design are normally distributed. Last assumption is that the variances in each of the group are not different from each other. Those assumptions can be verified using the residual analysis. A check of the normality assumption may be made by constructing a normal probability plot of the residuals. The normal probability plot of the residuals is illustrated in Fig. 3. If the residuals plot approximately along a straight line, then the normality assumption is satisfied[14].

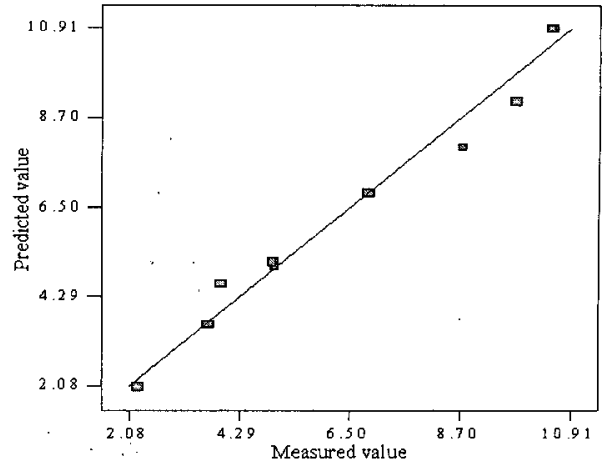

Fig. 2. The modeling result with the values between the predicted and the measured response.

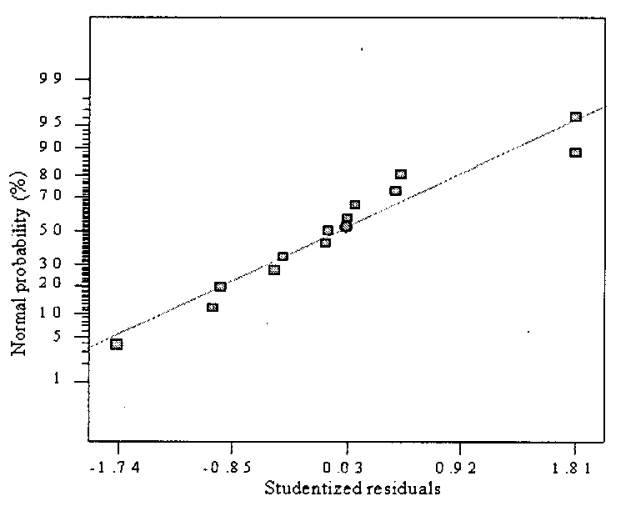

Fig. 3. The normal plot of residuals.

\section{RESULTS AND DISCUSSION}

The pretilt angle is measured by the crystal rotation method such as Fig. 4. Figure 4 shows the pretilt angle according to the rubbing strength when the hard baking temperature factor is fixed in middle point. Fig. 4(a), Fig 4(b) and Fig. 4(c) shows the pretilt angle when the rubbing strength is $0.3 \mathrm{~mm}, 0.5 \mathrm{~mm}$ and $0.7 \mathrm{~mm}$, respectively. The error rate of the result is approximately 0.002 .

The main effect plots for the response are illustrated in Fig. 5. The square dots are predicted response and the circular dots are measured response. They are alike in response. When the hard baking temperature factor is fixed in middle point, the effect of the rubbing strength plots for the pretilt angle is illustrated in Fig. 5(a). When the rubbing strength factor is fixed in middle point, the effect of the hard baking temperature plots for the pretilt angle is illustrated in Fig. 5(b). Figure 5(a) and Fig. 5(b) show that the pretilt angle is decreased when the rubbing strength or the hard baking temperature is increased. 


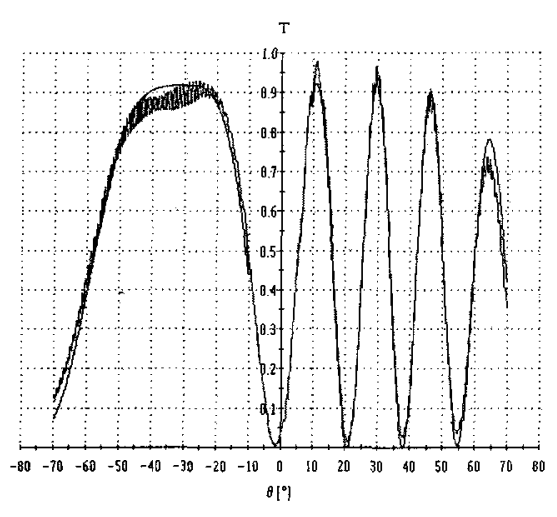

(a) $\mathrm{RS}=0.3 \mathrm{~mm}$

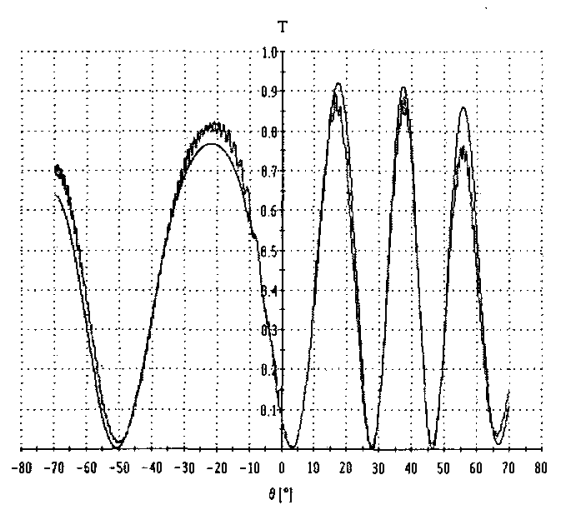

(b) $\mathrm{RS}=0.5 \mathrm{~mm}$

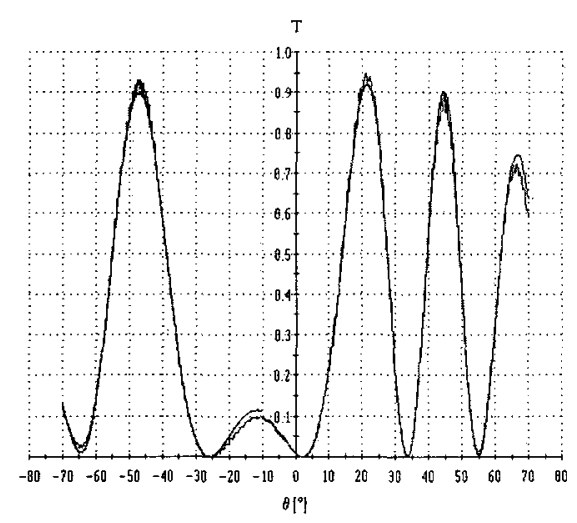

(c) $\mathrm{RS}=0.7 \mathrm{~mm}$

Fig. 4. The pretilt angle according to the rubbing strength.

Figure 5(a) shows that the pretilt angle on the PI surface in the middle hard baking temperature tends to increase as the rubbing strength increases. The rubbing treatment generates the obliquely inclined force on the alignment layer. Therefore, It seems quiet probable that the generation of the pretilt angle caused by a microasymmetrical triangular structure of the PI. The rubbing treatment is also attributed to the micro-roughness of the PI surface.

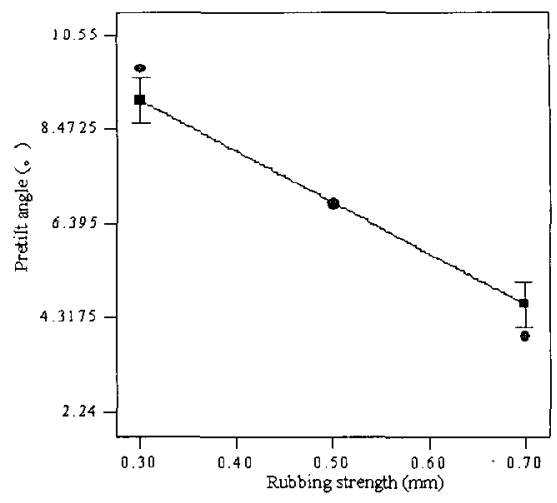

(a) The rubbing strength

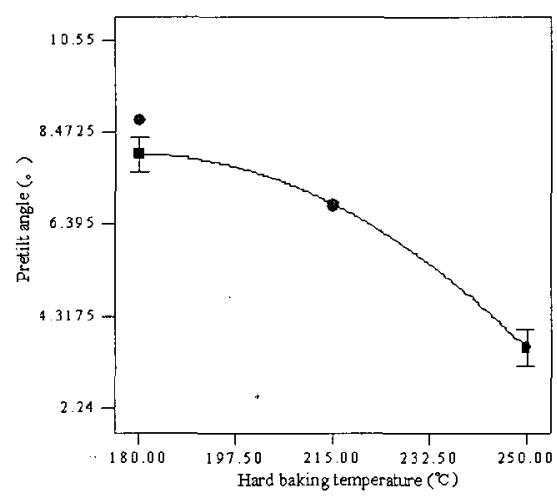

(b) The hard baking temperature

Fig. 5. The main effect plots for the pretilt angle generation.

The curve of Fig. 5(b) is caused by the square effect of the hard baking temperature. It shows that the pretilt angle on the PI surface in the middle rubbing strength tends to increase as the hard baking temperature increases. In the cases of the hard baking temperature at $180^{\circ} \mathrm{C}, 220^{\circ} \mathrm{C}$, and $250^{\circ} \mathrm{C}$, PI exhibited imidization rates of $20 \%, 68 \%$ and $100 \%$ in the previous work[15]. In order to explain the pretilt angle as a function of hard baking temperature, we must refer to the imidization ratio. As the hard baking temperature is increased, the imidization ratio is increased. Added to this, the pretilt angle is stabilized. In other words, the stability of the pretilt angle is tantamount to that of the imidization ratio. This result implies that the imidization ratio is a potent influence on the generation of the pretilt angle.

The interaction effect plot between the rubbing strength and the hard baking temperature for the pretilt angle is illustrated in Fig. 6. It shows that the generation of the pretilt angle in NLC on the rubbed PI surface with different baking temperatures $\left(180 \sim 250{ }^{\circ} \mathrm{C}\right)$ as a function of the rubbing strength. The upper straight line is a case of the low hard baking temperature and the under straight line is a case of the high hard baking temperature 


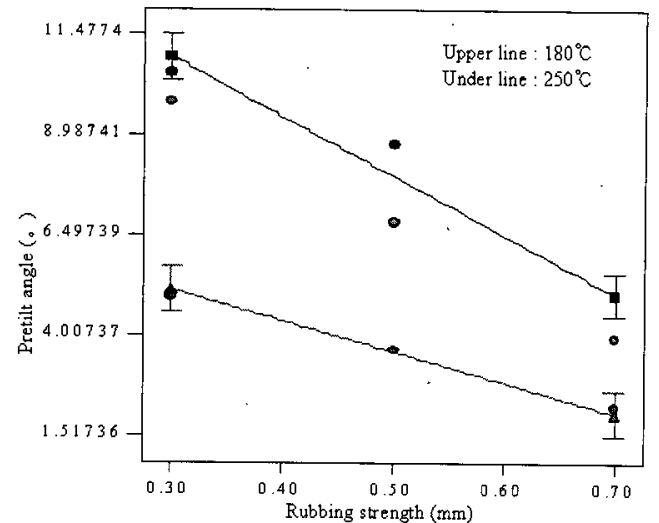

Fig. 6. The interaction effect plots for the pretilt angle.

The large difference between the effectiveness of one factor according to the other factor means that the interaction effect between the factors has a strong potential for the response. The rubbing strength is more effective for the pretilt angle when hard baking temperature is at the lower level than the higher level. It means that there is the difference of rubbing strength effect in accordance with the change of the hard baking temperature. This can be interpreted that the effect of the rubbing treatment is stronger when imidization rate is at the lower level. However, both the rubbing treatment and the hard baking temperature have a deep connection with the pretilt angle. The sum of the rubbing treatment and the hard baking temperature offers the key to the control of the pretilt angle.

The contour plot of the pretilt angle according to the change of the rubbing strength and the hard baking temperature is illustrated in Fig. 7. The smallest pretilt angle is generated applying the largest rubbing strength and highest hard baking temperature on the PI surfaces. In a similar fashion, the largest pretilt angle is generated applying the smallest rubbing strength and lowest hard baking temperature on the PI surfaces. As a consequence, it is very informative for the control of the pretilt angle. The contour plot of the pretilt angle according to the change of the rubbing strength and the hard baking temperature helps us to predict and realize the wanted pretilt angle. From the Fig. 3, we realize that contour plot can allow us to predict the pretilt angle with respect to the varying process conditions.

\section{CONCLURION}

The control of the pretilt angle in NLC on the homogeneous PI surface as a function of the rubbing strength and the baking temperature is investigated via the response surface modeling. The statistical significance factors are determined by ANOVA and those effects are

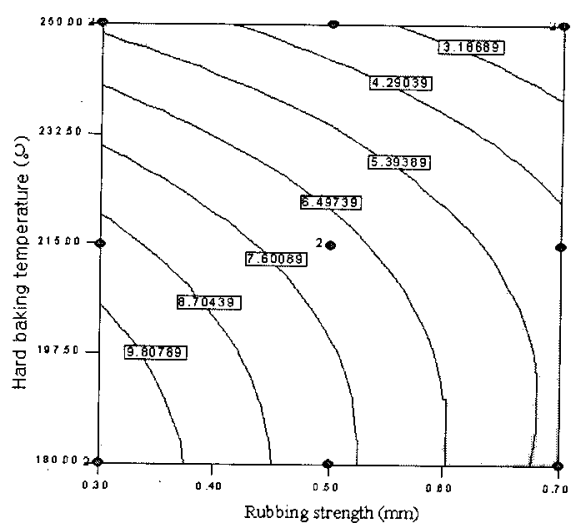

Fig. 7. The response surface plots for the pretilt angle.

compared with that of the varying process conditions, and are analyzed by the effect plots. The response surface modeling is in an agreement with the experimental data and represents a comprehensive characterization of the pretilt angle. From the results, the model will be able to allow us to predict the pretilt angle with respect to the varying process conditions. As a result, the statistical modeling of the control of the pretilt angle is very useful and gives reliability to predict the pretilt angle.

\section{REFERENCES}

[1] D.-S. Seo, N. Yoshida, S. Kobayashi, M. Nishikawa, and Y. Yabe, "Effects of conjugation of mesogenic core of nematic liquid crystals for polar anchoring energy and surface order parameter on rubbed polyimide film", Jpn. J. Appl. Phys., Vol. 33, No. 8B, p. L1174, 1994.

[2] Y. Iimura, S. Kobayashi, T. Hashimoto, T. Sugiyama, and K. Katoh, "Alignment control of liquid craytal molecules using photo-dimerization reaction of poly(vinyl cinnamate)", IEICE Trans. Electron, Vol. E79-C, No. 8, p. 1040, 1998.

[3] G. S. may, J. Huang, and C. J. Spanos, "Statistical experimental design in plasma etch modeling", IEEE Trans. Semiconduct. Manufact., Vol. 4, p. 83, 1991.

[4] L. K. Garling and G. P. Woods, "Enhancing the analysis of variance (ANOVA) technique with graphical analysis and its application to wafer processing equipment”, IEEE Trans. Comp., Hybrids, Manufact. Technol., Vol. 17, p. 149, 1994.

[5] J. H. Lau and C. Chang, "Taguchi design of experiment for wafer bumping by stencil printing", IEEE Trans. Electron. Packag. Manufact., Vol. 23, p. $219,2000$. 
[6] C. Hu, C. Tsay, and A. Bai, "Optimization of the hydrogen evolution activity on zinc-nickel deposits using experimental strategies", Electrochimica Acta, Vol. 48, p. 907, 2003.

[7] M. Yanaka, Y. Tsukahara, T. Okabe, and N. Takeda, "Statistical analysis of multiple cracking phenomenon of a SiOx thin film on a polymer substrate", J. Appl. Phys., Vol. 90, No. 2, p. 713, 2001.

[8] P. C. P. Bouten, "Failure test for brittle conductive layers on flexible display substrates", Eurodisplay, p. 313, 2002.

[9] D. R. Cairns, R. P. Witte II, D. K. Sparacin, S. M. Sachsman, D. C. Paine, G. P. Crawford, and R. R. Newton, "Strain-dependent electrical resistance of tin-doped indium oxide on polymer substrate", Appl. Phys. Lett., Vol. 76, No. 11, p. 1425, 2000.

[10] D.-S. Seo, K. Araya, N. Yoshida, M. Nishikawa, Y. Yabe, and S. Kobayashi, "Effect of the polymer tilt angle for generation of pretilt angle in nematic liquid crystal on rubbed polyimide surfaces", Jpn. J. Appl. Phys., Vol. 34, p. L503, 1995.
[11] D.-S. Seo, K. Muroi, and S. Kobayashi, "Generation of pretilt angle in nematic liquid crystal, 5CB, media aligned polyimide films prepared by spincoating and LB techniques : effect of rubbing", Mol. Cryst. Liq. Cryst., Vol. 213, p. 223, 1992.

[12] D.-S. Seo, S. Kobayashi, and M. Nishikawa, "Study of the pretilt angle for $5 \mathrm{CB}$ on rubbed polyimide films containing trifluoromethyl moiety and analysis of the surface atomic concentration of $\mathrm{F} / \mathrm{C}(\%)$ with an electron spectroscope for chemical analysis", Appl. Phys. Lett., Vol. 61, p. 2392, 1992.

[13] R. R. Hocking, "The analysis and selection of variables in linear regression", Biometrics, Vol. 32, p. $1,1976$.

[14] R. H. Myers and D. C. Montgomery, "Response surface methodology", Wiley inter. Sci., p. 42, 1995

[15] J.-Y. Hwang, K.-H. Nam, J.-H. Kim, D.-S. Seo, and D.-H. Suh, "Characterization and alignment properties on polyimide surface using polymer films for flexible liquid crystal displays", Jpn. J. Appl. Phys., Vol. 43, No. 12, p. 8179, 2004. 